\title{
Analysis of Electron Direct Tunneling Current through Very-Thin Gate Oxides in MOS Capacitors with the Parallel-Perpendicular Kinetic Energy Components and Anisotropic Masses
}

\author{
Fatimah Arofiati Noor, Mikrajuddin Abdullah, Sukirno, and Khairurrijal* \\ Physics of Electronic Materials Research Division, Faculty of Mathematics and Natural Sciences, \\ Institut Teknologi Bandung, Jalan Ganesa 10, Bandung 40132, Indonesia
}

(Received on 30 December, 2009)

\begin{abstract}
An electron direct tunneling current model of $n^{+}-$poly $-\mathrm{Si} / \mathrm{SiO}_{2} / \mathrm{p}-\mathrm{Si}(100)$ metal-oxide-semiconductor (MOS) capacitors has been developed by considering a parallel-perpendicular kinetic energy coupling, which is represented by the gate electron phase velocity, and anisotropic masses under a parabolic $E-k$ dispersion relationship. The electron effective mass in the oxide and the electron phase velocity in the $n^{+}$poly $-S i$ gate are the only two fitting parameters to compare calculated tunneling currents to measured ones. It was obtained that the calculated tunneling currents fit well to the measured ones. The electron effective mass in the oxide layer tends to increase with decreasing the oxide thickness. In addition, the gate electron velocity is a constant of $1 \times 10^{5} \mathrm{~m} / \mathrm{s}$. Moreover, the theoretical model offers a simple treatment and an accurate result in obtaining the tunneling current.
\end{abstract}

Keywords: gate oxide, anisotropic mass, gate velocity, tunneling current

\section{INTRODUCTION}

It is well known that a condition for the success of the semiconductor industry is the continuous improvement of integrated circuit performance by simply reducing the feature size of the fundamental switching components, metal-oxidesemiconductor field-effect transistors (MOSFETs) [1]. The miniaturization of device dimensions allows the high performance at low costs [2]. In order to reach that purpose, the oxide thickness is supposed to be shrunk to $1.5-1.9 \mathrm{~nm}$ when the gate length of MOSFETs is scaled down to $100 \mathrm{~nm}$ [3]. However, the tunneling current through very thin gate oxides increases exponentially as the oxide thickness decreases at low applied voltages, whereas the transistor performance is significantly enhanced [4]-[5]. In addition, the huge tunneling current in very large scale integrated circuits causes the power consumption becomes significantly high [6]. Therefore, it is required a precise model to foresee the tunneling current for developing future transistor and circuit design.

A theoretical model of tunneling current in the MOS capacitor had been originated since long year ago. Electron transmittance, tunneling time and current in MOS capacitors have been investigated under an isotropic mass approach and without considering a coupling effect between parallel and perpendicular motions by employing the parabolic $E-k$ dispersion relationship [5], [7]-[11]. However, the tunneling currents in the MOS capacitor fit well to the measured ones only for the oxide voltages less than $1.5 \mathrm{~V}$ and get significantly greater than the measured one at oxide voltages above $1.5 \mathrm{~V}$ [5]. The parabolic $E-k$ dispersion relationship becomes commonly used to solve the tunneling problems due to its simplicity. It is known that the non-parabolic $E$ - $k$ dispersion relationship is more accurate than the parabolic $E-k$ dispersion relationship [5]. However, the non-parabolic $E-k$ dispersion relationship is more complicated. Therefore, it is necessary to find the simple and accurate method in describing the tunneling current.

${ }^{*}$ Electronic address: krijalefi.itb.ac.id
In this paper, we report a theoretical model of tunneling current in $n^{+}-$poly $-\mathrm{Si} / \mathrm{SiO}_{2} / p-\mathrm{Si}(100)$ structures under an anisotropic mass approach and taking into account a parallel-perpendicular kinetic energy components which is represented by the gate electron phase velocity by using the parabolic $E-k$ dispersion relationship. The electron effective mass in the oxide layer and the electron phase velocity in the $n^{+}-$poly - Si gate, which are two fitting parameters used to fit the theoretical tunneling currents to the measured ones, will be discussed.

\section{THEORETICAL MODEL}

Figure 1 describes a schematic energy diagram of $n^{+}-$ poly $-\mathrm{Si} / \mathrm{SiO}_{2} / \mathrm{p}-\mathrm{Si}(100)$ structures at zero bias and a negative bias applied to the $n^{+}-$poly $-S i$ gate. Here, the barrier width and height are $t_{o x}$ and $\phi_{B}$, respectively, $V_{o x}$ is the oxide voltage, and $e$ is the electronic charge.

Using a simple electrostatic analysis, the potential profile shown in Figure 1.(b) is mathematically expressed as

$$
V(z)=\left\{\begin{array}{cc}
0 & z<0 \\
\phi_{\mathrm{B}}-e F z & 0 \leq z<t_{o x} \\
-e V_{o x} & z \geq t_{o x}
\end{array}\right.
$$

where $F=\frac{e V_{o x}}{t_{o x}}$ is the electric field in the barrier.

An electron in an anisotropic material under the parabolicband effective mass approximation is given by [12] :

$$
\left(\frac{1}{2} \boldsymbol{p}^{T} \frac{\alpha(\boldsymbol{r})}{m_{0}} \boldsymbol{p}+V(\boldsymbol{r})\right) \Psi(\boldsymbol{r})=E \Psi(\boldsymbol{r}),
$$

where $m_{0}$ is the free-electron mass, $\boldsymbol{p}$ is the momentum vector, $\alpha(\boldsymbol{r}) / m_{0}$ is the inverse effective-mass tensor, $V(\boldsymbol{r})$ is the potential energy, and $E$ is the total energy. Since the potential energy is only dependent on the $z$ direction, the electron wavefunction $\Psi(\boldsymbol{r})$ is given by $\Psi(\boldsymbol{r})=$ $\psi(z) \exp (-i \gamma z) \exp \left(i\left(k_{x} x+k_{y} y\right)\right)$, where $k_{x}$ and $k_{y}$ are the wavenumbers in the $x$ - and $y$-directions, respectively. The term $\gamma=\left(\alpha_{x z} k_{x}+\alpha_{y z} k_{y}\right) / \alpha_{z z}$ is the wavenumber in the $x-y$ plane and $\alpha_{i j}$ is the tensor element with $i, j \in\{x, y, z\}$. 


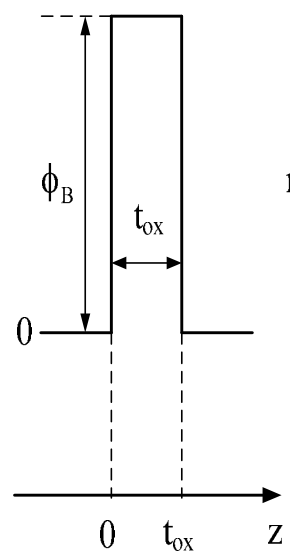

(a)

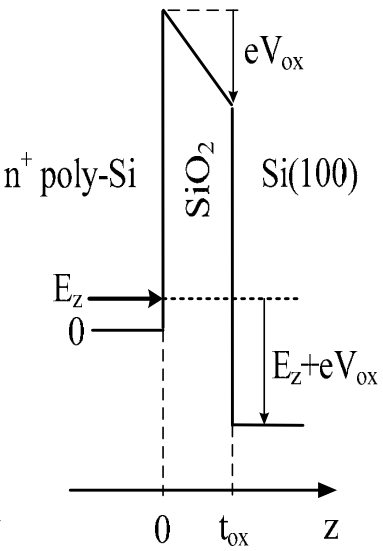

(b)
FIG. 1: Energy band profile of a $n^{+}-$poly $-\mathrm{Si} / \mathrm{SiO}_{2} / p-\mathrm{Si}(100)$ structure without applied voltage (a) and a negative bias applied to the $n^{+}-$poly - Si gate (b).

Since the total energy $E$ comprises the parallel (the $x-y$ plane) and perpendicular (the $z$-direction) energies, the wavefunction in the $z$-direction $\psi(z)$ fulfills the one-dimensional Schrödinger-like equation that is written as

$$
-\frac{\hbar^{2}}{2 m_{0}} \alpha_{z z} \frac{d^{2} \psi(z)}{d z^{2}}+V(z) \psi(z)=E_{z} \psi(z),
$$

where $\hbar$ is the reduced Planck constant. The perpendicular energy $E_{z}$ is then written as $E_{z}=E-\sum_{i, j \in\{x, y\}} \frac{\hbar^{2}}{2 m_{0}} \beta_{i j} k_{i} k_{j}$, where $\beta_{i j}=\alpha_{i j}-\left(\alpha_{i z} \alpha_{z j} / \alpha_{z z}\right)$.

Referring to Figure 1.(b) and considering that the total energy of electron is constant, the perpendicular energy in region $n(n \in\{2,3\})$ with respect to that in region 1 is

$$
E_{z, n}=E_{z, 1}+\frac{\hbar^{2}}{2 m_{0}} \sum_{i, j \in\{x, y\}}\left(\beta_{i j, 1}-\beta_{i j, n}\right) k_{i} k_{j} .
$$

By substituting Equation (4) into Equation (3), the Schrödinger-like equation in region $n$ is found as

$$
-\frac{\hbar^{2}}{2 m_{0}} \alpha_{z z, n} \frac{d^{2} \psi(z)}{d z^{2}}+V_{e f f}(z) \psi(z)=E_{z, 1} \psi(z) .
$$

Equation (5) contains the coupling between parallel and perpendicular kinetic energy components of the electron motion in an anisotropic MOS capacitor. The effective potential en$\operatorname{ergy} V_{e f f}(z)$ is written as

$$
V_{e f f}(z)=V(z)-\sum_{i, j \in\{x, y\}} \frac{v_{e}^{2} m_{0}}{2 \beta_{i j, 1}}\left(1-\frac{\beta_{i j, n}}{\beta_{i j, 1}}\right),
$$

where $v_{e}$ is the gate electron phase velocity. This effective potential energy occurs due to the difference of parallel kinetic energies between regions $n$ and 1 due to the difference between the effective masses in the $x-y$ plane of regions $n$ and 1. A complete derivation can be found in Ref. [13].

The electron wavefunction in the $z$-direction $\Psi(z)=$ $\psi(z) \exp (-i \gamma z)$ in each region is given by

$$
\Psi(z)=\left\{\begin{array}{cc}
\left\{\mu \exp \left(i k_{1} z\right)+\eta \exp \left(-i k_{1} z\right)\right\} \exp \left(-i \gamma_{1} z\right) & z<0 \\
\{\lambda A i(\varepsilon(z))+\delta B i(\varepsilon(z))\} \exp \left(-i \gamma_{2} z\right) & 0 \leq z<t_{o x} \\
\left\{\chi \exp \left(i k_{3} z\right)\right\} \exp \left(-i \gamma_{3} z\right) & z \geq t_{o x}
\end{array}\right.
$$

Here, $\mu, \eta, \lambda, \delta$, and $\chi$ are constants, and $k_{1}$ and $k_{3}$ are the wavenumbers in the $z$-direction in regions 1 and 3 , respectively, and expressed as

$$
\begin{aligned}
& k_{1}=\left(\frac{2 m_{0} E_{z}}{\hbar^{2}} \frac{1}{\alpha_{z z, 1}}\right)^{1 / 2}, \\
& k_{3}=\left[\frac { 1 } { \alpha _ { z z , 3 } } \left\{\frac{2 m_{0}\left(E_{z}+e V_{o x}\right)}{\hbar^{2}}\right.\right. \\
& \left.\left.+\sum_{i, j \in\{x, y\}} \frac{v_{e}^{2} m_{0}^{2}}{\hbar^{2}} \frac{1}{\beta_{i j, 1}}\left(1-\frac{\beta_{i j, 3}}{\beta_{i j, 1}}\right)\right\}\right]^{1 / 2} .
\end{aligned}
$$

The arguments $\varepsilon(z)$ of the Airy functions $A i$ and $B i$ in
Equation (7) is

$$
\begin{aligned}
& \varepsilon(z)=\left(\frac{2 m_{0}}{\hbar^{2}} \frac{1}{\alpha_{z z, 2}} F\right)^{1 / 3} \\
& \left(-z+\frac{\left(\phi_{B}-E_{z}\right)}{F}-\sum_{i, j \in\{x, y\}} \frac{v_{e}^{2} m_{0}}{2 F} \frac{1}{\beta_{i j, 1}}\left(1-\frac{\beta_{i j, 2}}{\beta_{i j, 1}}\right)\right) .
\end{aligned}
$$

By applying the boundary condition at each interface [14], the electron transmittance through the barrier is easily obtained as given in Refs. [15]-[16]. The obtained electron transmittance is then utilized to calculate the tunneling cur- 


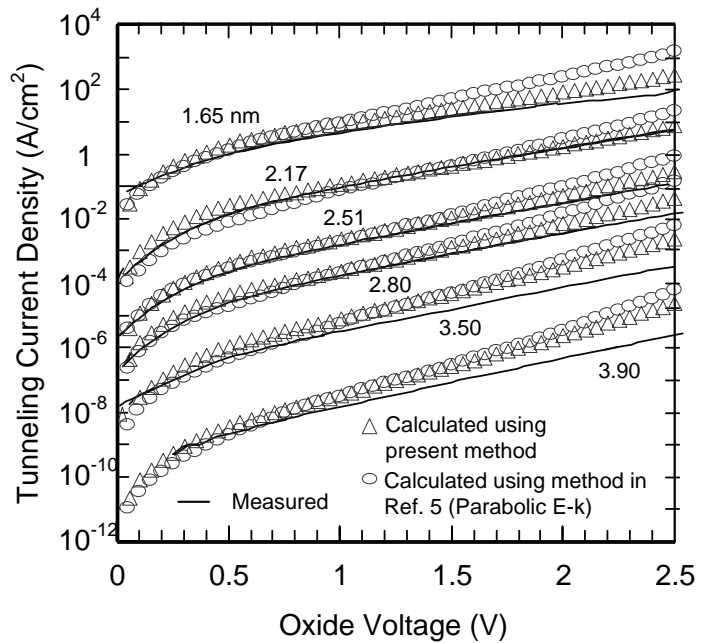

FIG. 2: Measured and calculated tunneling current density through 1.65-2.90-nm-thick oxides using the present and previous method under parabolic $E-k$ dispersion relationships. The previous method does not include the parallel-perpendicular kinetic energy coupling and anisotropic masses.

rent by using the following equation [5]:

$$
\begin{aligned}
& J_{z}=\frac{e \sum_{l} n_{v l} m_{d l}}{2 \pi^{2} \hbar^{3}} \\
& \times \int_{0}^{\infty} T\left(E_{z}\right) k T \ln \left\{\frac{1+\exp \left[\left(E_{F}-E_{z}\right) / k T\right]}{1+\exp \left[\left(E_{F}-E_{z}-e V_{o x}\right) / k T\right]}\right\} d E_{z},
\end{aligned}
$$

where $l$ is the number of valley, $n_{v}$ and $m_{d}$ are the valley degeneracy and the density-of-states mass per valley, respectively, $T\left(E_{z}\right)$ is the electron transmittance, $E_{F}$ is the Fermi energy of the gate, $k$ is the Boltzmann constant, and $T$ is the temperature. The tunneling current in Equation (11) is easily calculated by employing the Gauss-Laguerre Quadrature method [17].

\section{CALCULATED RESULTS AND DISCUSSION}

There are six equivalent valleys in the conduction band of $\operatorname{Si}(100)$, which are divided into three groups of valleys with the tensor elements $\alpha_{i j}$ as shown in Table 1 [18]-[20]. The electron effective mass in the oxide layer, $m_{o x}$, and the phase velocity of an injected electron from the $n^{+}-$poly$S i$ gate, $v_{e}$, are the only two fitting parameters to compare the measured tunneling current with the theoretical one. In order to verify the present model, we used all parameters and measured current densities from Ref. [5].

Figure 2 shows the calculated tunneling currents obtained using the previous method under parabolic $E-k$ dispersion relationships fit to the measured ones only in the low oxide voltage regime. Moreover, the calculated tunneling currents employing the present method reveals better fitting to the measured ones. This means that the present method including the parallel-perpendicular kinetic energy coupling and

\begin{tabular}{cccc}
\hline Valley & Si(100) \\
\hline V1 & 1.02 & 0 & 0 \\
& 0 & 5.26 & 0 \\
& 0 & 0 & 5.26 \\
\hline V2 & 5.26 & 0 & 0 \\
& 0 & 1.02 & 0 \\
\hline & 0 & 0 & 0.26 \\
\hline V3 & 5.26 & 0 & 0 \\
& 0 & 5.26 & 1.02 \\
\hline & 0 & 0 &
\end{tabular}

anisotropic masses offers more accurate calculations for tunneling current in particular at high voltage regimes. For all oxide thicknesses, the fitted value of the injected electron velocity $v_{e}$ is $1 \times 10^{5} \mathrm{~m} / \mathrm{s}$, independent of the oxide thickness.

Figure 3 depicts the measured and calculated tunneling currents obtained using the present method under anisotropic masses and considering a coupling between parallel and perpendicular motions by employing the parabolic $E-k$ dispersion relationship, and the previous method without including the parallel-perpendicular kinetic energy coupling and anisotropic masses by using the non-parabolic $E$ - $k$ dispersion relationship. It is shown that the obtained tunneling currents under present method are almost the same as those obtained under the previous method. Noting that the non-parabolic $E$ $k$ dispersion relationship is more accurate and complicated than the parabolic $E-k$ dispersion relationship as given in Ref. [5], the present model gives the simple treatment and accurate results in calculating the tunneling current.

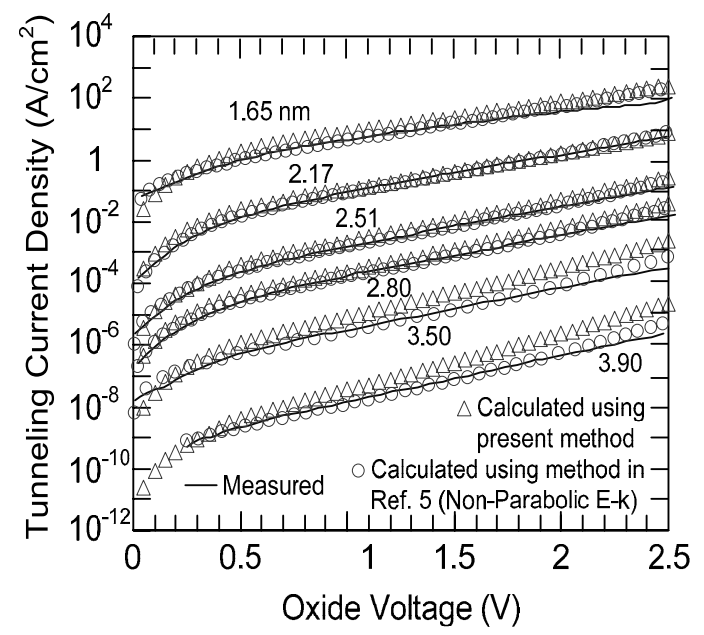

FIG. 3: Measured and calculated tunneling currents using the present method by employing the parabolic $E-k$ dispersion relationship, and the previous method by using the non-parabolic $E-k$ dispersion relationship without including the parallel-perpendicular kinetic energy coupling and anisotropic masses.

As depicted in Figure 4 the fitted value of $m_{o x}$ for $t_{o x}$ 
thicker than $3.5 \mathrm{~nm}$ is consistent with that obtained by Khairurrijal, et al. under parabolic $E-k$ dispersion relationships where $m_{o x}=0.4-0.42 m_{0}$ [5]. However, for $t_{o x}$ thinner than $2.8 \mathrm{~nm}, m_{o x}$ alters from 0.52 to $0.78 m_{0}$ as $t_{o x}$ decreases to $1.65 \mathrm{~nm}$. These imply that $m_{o x}$ tends to enhance as $t_{o x}$ reduces. The fitted value of electron effective mass $m_{o x}$ calculated under the present method differs insignificantly with that obtained under the previous method by using the parabolic $E-k$ dispersion relationship. It means that the present model does not change significantly the fitted effective mass obtained from the previous model. It is shown that the inclusion of the coupling effect represented by the electron phase velocity in the gate to the present model offers better fitting process of tunneling currents especially at high voltage regimes with the simple treatment.

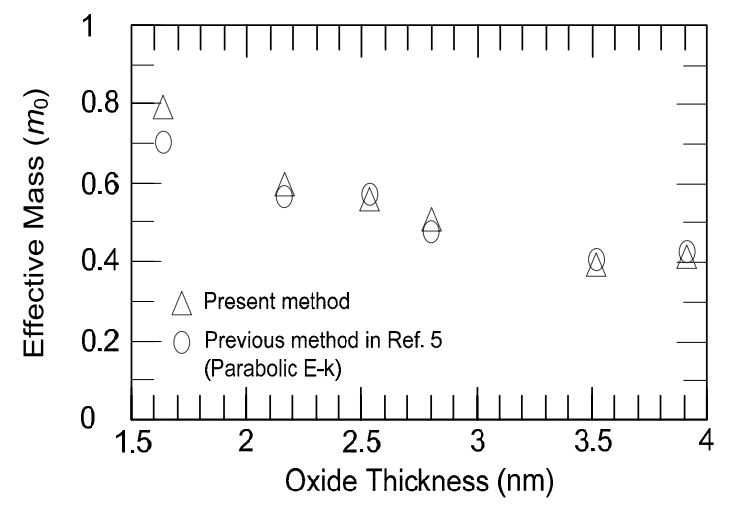

FIG. 4: Fitted value of the electron effective mass $m_{o x}$ calculated using previous and present models.

\section{CONCLUSIONS}

We have studied theoretically the electron tunneling current density in the $n^{+}-$poly $-\mathrm{Si} / \mathrm{SiO}_{2} / p-\mathrm{Si}(100)$ MOS capacitor. The transmittance through the barrier is derived by including the effects of a parallel-perpendicular kinetic energy coupling and anisotropic masses represented by the gate electron phase velocity under parabolic $E-k$ dispersion relationships. It has been shown that the measured tunneling current densities are well fitted to the calculated ones by utilizing the electron effective mass in the oxide layer and the gate electron phase velocity as fitting parameters. For oxide thickness thicker than $3.5 \mathrm{~nm}$, the fitted value of the electron effective mass is consistent to that used in previous model in which the coupling between parallel and perpendicular kinetic energies (the gate electron phase velocity) is not taken into account. It has been found that the electron effective mass in the oxide layer tends to enhance with reducing the oxide thickness. In addition, the gate electron phase velocity is $1 \times 10^{5} \mathrm{~m} / \mathrm{s}$ independent of the oxide thickness. Furthermore the present model is capable of elucidating the important role of the coupling effect to obtain tunneling currents with the accurate result and simple treatment.

\section{Acknowledgment}

One of the authors (F.A.N) would like to thank the Habibie Center Foundation, Indonesia, for the domestic doctoral scholarship at Institut Teknologi Bandung.
[1] Y. Kamata, Materials Today 11, 30 (2008)

[2] V. K. Khanna, Phys. Rep. 398, 67 (2004).

[3] Khairurrijal, W. Mizubayashi, S. Miyazaki, and M. Hirose, Appl. Phys. Lett. 77, 3580 (2000).

[4] H. S. Momose, M. Ono, T. Yoshitomo, T. Ohguro, S. Nakamura, M. Saito, and H. Iwai, IEEE Trans. Electron Devices ED-43, 1233 (1996).

[5] Khairurrijal, W. Mizubayashi, S. Miyazaki, and M. Hirose, J. Appl. Phys. 87, 3000 (2000).

[6] S.-H. Lo, D. A. Buchanan, Y. Taur, and W. Wang, IEEE Electron Device Lett. 18, 209 (1997).

[7] Khairurrijal, F. A. Noor, and Sukirno, Solid-State Electron. 49, 923 (2005).

[8] F. A. Noor, M. Abdullah, Sukirno, and Khairurrijal, Indonesian J. Phys. 18, 41 (2007).

[9] L. Larcher, IEEE Trans. Electron Devices 48, 271 (2001).

[10] B. Govoreanu, P. Blomme, M. Rosmeulen, J. Van Houdt, and K. De Meyer, Solid-State Electron. 47, 1045 (2003).

[11] E. M. Vogel, K. Z. Ahmed, B. Hornung, W. K. Henson, P. K.
McLarty, G. Lucovsky, J. R. Hauser, and J. J. Wortman, IEEE Trans. Electron Devices 45, 1350 (1998).

[12] K.-Y. Kim and B. Lee, Phys. Rev. B 58, 6728 (1998).

[13] L. Hasanah, M. Abdullah, Sukirno, T. Winata, and Khairurrijal, Semicond. Sci. Technol. 23, 125024 (2008).

[14] K.-Y. Kim and B. Lee, Superlattices Microstruct. 24, 389 (1998).

[15] Khairurrijal, S. Miyazaki, and M. Hirose, J. Vac. Sci. Technol. B 17, 306 (1999).

[16] F. A. Noor, M. Abdullah, Sukirno, and Khairurrijal, Indonesian J. Phys. 18, 1 (2007).

[17] P. L. De Vries, A First Course in Computational Physics, New York: Wiley (1993) 173.

[18] K. S. Yi and J. J. Quinn, Phys. Rev. B 27, 1184 (1983).

[19] K. S. Yi and J. J. Quinn, Phys. Rev. B 27, 2396 (1983).

[20] A. Rahman, M. S. Lundstrom, and A. W. Ghosh, J. Appl. Phys. 97, 053702 (2005). 